\title{
Impact of chitosan membrane culture on the expression of pro- and anti-inflammatory cytokines in mesenchymal stem cells
}

\author{
PENG YI ${ }^{*}$, XIONGFENG XU*, BO QIU and HUAJIE LI \\ Department of Orthopedic Surgery, Renmin Hospital of Wuhan University, Wuhan, Hubei 430060, P.R. China
}

Received August 5, 2019; Accepted July 17, 2020

DOI: $10.3892 /$ etm.2020.9108

\begin{abstract}
Osteoarthritis (OA) is a chronic inflammatory joint condition caused by various inflammatory cytokines. The pro-inflammatory cytokines controlling OA include interleukin (IL)-1 $\beta$, tumor necrosis factor (TNF)- $\alpha$, IL-6 and IL-18. The anti-inflammatory cytokines include IL-4, IL-10, IL-13, leukemia inhibitory factor (LIF), glycoprotein 130 (IL6ST), TNF- $\alpha$-stimulated gene 6 and transforming growth factor (TGF)- $\beta 1$. Mesenchymal stem cells (MSCs) serve an anti-inflammatory role in the treatment of OA by secreting various cytokines. Previous studies demonstrated that the anti-inflammatory ability of MSCs decreased rapidly in a traditional plate culture. Maintaining the anti-inflammatory properties of MSCs in vitro remains challenging. Therefore, it is necessary to develop a more stable and efficient method to culture MSCs in vitro. Chitosan is a deacetylated derivative of chitin and is the second most abundant natural polysaccharide worldwide. The present study demonstrated that that MSCs cultured on chitosan membranes (CM) spontaneously formed multicellular spheroids. Compared with the control group without CM, the formation of multicellular spheres in the CM enhanced the anti-inflammatory properties of MSCs. Expression levels of pro- and anti-inflammatory genes mRNA and their proteins in MSCs were detected by reverse transcription-quantitative PCR, western blot analysis and immunofluorescence assay. Protein and mRNA expression levels of pro-inflammatory cytokines IL-1 $\beta$, TNF- $\alpha$, IL-6 and IL-18 were significantly decreased in CM-cultured MSCs compared with the control group $(\mathrm{P}<0.05)$. Furthermore, mRNA and protein expression levels of anti-inflammatory cytokines TGF- $\beta 1$ in CM-cultured MSCs were significantly
\end{abstract}

Correspondence to: Professor Bo Qiu, Department of Orthopedic Surgery, Renmin Hospital of Wuhan University, 238 Liberation Road, Wuhan, Hubei 430060, P.R. China

E-mail: qbtg163@163.com

*Contributed equally

Key words: chitosan membrane, mesenchymal stem cells, multicellular spheroids, pro-inflammatory cytokines, anti-inflammatory cytokines, osteoarthritis increased compared with the control group $(\mathrm{P}<0.01)$. These results indicated that the formation of multicellular spheroids by CM-cultured MSCs aided in maintaining anti-inflammatory effects.

\section{Introduction}

Osteoarthritis (OA) is a disease that causes articular cartilage degeneration, and there is currently no effective drug to prevent disease progression (1). Medical care is mainly based on alleviating painful symptoms (2). The pathological changes in OA involve all tissues that form joints. During the development of OA, intra-articular tissues exhibit inflammation of varying severity, and joint inflammation may cause joint destruction. It is hypothesized that the immune system is one of the factors involved in the pathogenesis, occurrence and development of OA (3). During OA development, the production and function of cytokines vary according to the duration and severity of the disease (4). Cytokines can be divided into pro- and anti-inflammatory according to their roles in OA (3). Cytokines interfere with the process of catabolism and anabolism, particularly in tissues that are often subjected to high mechanical loads, such as the human joints. The imbalance between synthesis and catabolism causes progressive degeneration of the articular cartilage, causing gradual development of the disease (5).

Mesenchymal stem cells (MSCs) are adult stem cells derived from the multi-directional differentiation potential of tissues and organs and can differentiate into osteogenic (6), cartilage (7) and liver cells (8). Previous studies have demonstrated that MSCs can be transformed into chondrocytes and induced into articular cartilage to repair damage, and have therapeutic effects on experimental OA $(9,10)$. Currently, two-dimensional cultures cannot maintain the stability of MSCs. This is primarily due to the senescence of MSCs during the process of a traditional plate culture, self-differentiation of osteoblasts, decrease in anti-inflammatory ability and decline in proliferation ability which severely affect the function of MSCs as a cell therapy (11). Previous studies have reported that aggregation of MSCs into 3-dimensional (3D) spheroids could markedly enhance their trophic and anti-inflammatory properties $(11,12)$.

Chitosan (CS) is a deacetylated derivative of chitin and is the second most abundant natural polysaccharide in the world (13). The biocompatibility of CS has been attributed to 
its structural and functional similarity to glycosaminoglycans, making it a biomaterial candidate for cartilage engineering (14). In the present study, MSCs were cultured on CS membranes (CMs) to form 3D multicellular spheres. The formation of multicellular spheres had a significant effect on the expression of anti-inflammatory genes in MSCs. The aim of the present study was to investigate the impact of a $\mathrm{CM}$ cultures on the expression of pro- and anti-inflammatory cytokines in human umbilical cord MSCs and to establish a foundation for further study on the role of CM-cultured MSCs in OA cartilage repair.

\section{Materials and methods}

Experimental materials. CS powder (Sinopharm Group Chemical Reagent Co., Ltd.), FBS (Gibco; Thermo Fisher Scientific, Inc.), DMEM (Gibco; Thermo Fisher Scientific, Inc.), a SDS-PAGE gel preparation kit (cat. no. AS1012; Aspen), RIPA total protein lysis and extraction buffer (cat. no. AS1004; Aspen), a BCA protein concentration assay kit (cat. no. AS1086; Aspen), protein markers (Thermo Fisher Scientific, Inc.), a fluorescence quantitative PCR instrument (Thermo Fisher Scientific, Inc.), TRIzol ${ }^{\circledR}$ reagent (Invitrogen; Thermo Fisher Scientific, Inc.), SYBR ${ }^{\circledR}$ Premix Ex Taq ${ }^{\mathrm{TM}}$ (Takara Biomedical Technology, Co., Ltd.), PrimeScript ${ }^{\mathrm{TM}}$ RT reagent kit with gDNA Eraser (Takara Biomedical Technology, Co., Ltd.), PCR primers (Wuhan Google Biotechnology Company Co., Ltd.), trypsin EDTA (Gino Biomedical Technology Co., Ltd.) and PBS (Gino Biomedical Technology Co., Ltd) were used in the present study.

Preparation of the CM. CS powder was dissolved in $1 \%$ glacial acetic acid solution(Sinopharm Group Chemical Reagent Co., Ltd.) to obtain a $1 \%$ CS solution, which was spread evenly at the bottom of a 6 -well culture plate at $1.2 \mathrm{ml} /$ well. The liquid was dried in an oven at $65^{\circ} \mathrm{C}$ for $24 \mathrm{~h}$ to prepare a CM substrate. The CM substrate was exposed to ultraviolet light overnight, neutralized using a $0.5 \mathrm{~mol} / 1 \mathrm{NaOH}$ solution for $10 \mathrm{~min}$ at room temperature. Lye residue was thoroughly washed with sterile water three times. The CM substrate was subsequently washed with PBS three times.

Cultivation of primary MSCs. The present study was approved by the Ethics Committee of the Renmin Hospital of Wuhan University (Wuhan, China) and written informed consent. Umbilical cords were obtained from three healthy postpartum women (age, Patient 1, 28 years; Patient 2, 28 years; and Patient 3, 30 years) in May 2017. Umbilical cords were collected and washed thoroughly with PBS containing $100 \mu \mathrm{g} / \mathrm{ml}$ streptomycin and $100 \mathrm{U} / \mathrm{ml}$ penicillin. Wharton's jelly tissue was separated from the umbilical cords with forceps, cut into smaller pieces with scissors and digested with $0.1 \%$ type I collagenase (Invitrogen; Thermo Fisher Scientific, Inc.) at $37^{\circ} \mathrm{C}$ for $16 \mathrm{~h}$ to release MSCs. MSCs were obtained following by centrifugation for $5 \mathrm{~min}$ at $400 \mathrm{x} \mathrm{g}$ at room temperature and cultured in high glucose MEM/F12 (Invitrogen; Thermo Fisher Scientific, Inc.) supplemented with 10\% FBS (Hyclone; GE Healthcare Life Sciences) and $10 \mathrm{ng} / \mathrm{ml}$ basic fibroblast growth factor (Peprotech EC Ltd.). The medium was replaced every 3 days with fresh medium.
MSCs were identified by morphological and flow cytometric analyses using an inverted microscope at a magnification of x40 and x100 (Olympus Corporation). When their proliferation reached $80 \%$, the $\mathrm{P} 1$ generation was used as seed cells.

Experimental grouping and cell culture. The following groups were used in the present study: i) The experimental group, in which CM covered the bottom of the 6-well plate; and ii) the control group, in which the 6-well plate did not undergo treatment. MSCs P1 cells were inoculated into the groups (seeding density, $3 \times 10^{5} /$ well) in a low-sugar DMEM medium containing $10 \% \mathrm{FBS}$ in $\mathrm{a} \mathrm{CO}_{2}$ incubator at $37^{\circ} \mathrm{C}$. At $72 \mathrm{~h}$ post-inoculation, cell morphologies were observed using an inverted microscope at a magnification of $\mathrm{x} 40$ and $\mathrm{x} 100$ (Olympus Corporation) and cells were collected for subsequent experiments.

Reverse transcription-quantitative PCR (RT-qPCR). Expression levels of pro- and anti-inflammatory genes in MSCs cultured in the experimental and control groups were detected using RT-qPCR. Total RNA was extracted from the two groups of cells cultured for $72 \mathrm{~h}$ and transcribed into cDNA using a reverse transcriptase kit. A total of $2 \mu \mathrm{l}$ cDNA was used as a PCR amplification template. $\beta$-actin was used as the internal reference gene. All qPCR reactions were performed using the following thermocycling conditions: Initial denaturation at $95^{\circ} \mathrm{C}$ for $30 \mathrm{sec}$, followed by 40 cycles of $95^{\circ} \mathrm{C}$ for $5 \mathrm{sec}, 58^{\circ} \mathrm{C}$ for $30 \mathrm{sec}$ and $72^{\circ} \mathrm{C}$ for $30 \mathrm{sec}$. SYBR ${ }^{\circledR}$ Premix Ex Taq ${ }^{\mathrm{TM}}$ (Takara Biomedical Technology, Co., Ltd.) was used for the quantitative analysis using a PCR instrument. The $2^{-\Delta \Delta C q}$ method was used to analyze results (15). Primer sequences are presented in Table I.

Western blotting. Expression levels of pro- and anti-inflammatory cytokines in MSCs were detected using western blotting. MSCs in the experimental and control groups were lysed with pre-cooled RIPA buffer for $30 \mathrm{~min}$ and centrifuged at $13,000 \times \mathrm{g}$ for $5 \mathrm{~min}$ at $4^{\circ} \mathrm{C}$. The supernatant was collected and total protein amounts were measured using a BCA kit (Beyotime Institute of Biotechnology). A total of $20 \mu \mathrm{g}$ total protein/lane was electrophoresed on SDS-PAGE (10\% gel) and transferred to $0.22-\mu \mathrm{m}$ PVDF membranes. Membranes were blocked with $5 \%$ skim milk TBST $(10 \mathrm{mmol} / \mathrm{l}$ Tri-HCl; $150 \mathrm{mmol} / \mathrm{l} \mathrm{NaCl} ; 0.25 \%$ Tween-20; $\mathrm{pH} 7.5)$ for $1 \mathrm{~h}$ at room temperature. Membranes were then incubated with polyclonal rabbit anti-human $\beta$-actin (1:10,000; cat. no. TDY051; BEIJING TDY BIOTECH CO.,LTD.), interleukin (IL)- $1 \beta$ (1:1000; cat. no. ab2105; Abcam), tumor necrosis factor- $\alpha$ (TNF- $\alpha$; 1:500; cat. no. ab66579; Abcam), IL-6 (1:1000; cat. no. 21865-1-AP; Proteintech Group, Inc), IL-18 (1:1000; cat. no. 10663-1-AP; Proteintech Group, Inc), IL-4 (1:1000; cat. no. ab9622; Abcam), IL-10 (1:1000; cat. no. DF6894; affbiotech), IL-13 (1:500; cat. no. ab106732; Abcam), leukemia inhibitory factor (LIF; 1:1000; cat. no. ab113262; Abcam), glycoprotein 130 (IL6ST; 1:500; cat. no. ab202850; Abcam), TNF- $\alpha$-stimulated gene 6 (TSG6; 1:500; cat. no. ab128266; Abcam), transforming growth factor (TGF) $\beta 1$ (1:1000; cat. no. AF1027; affbiotech) overnight at $4^{\circ} \mathrm{C}$. Membranes were washed with TBST and incubated with horseradish peroxidase-conjugated goat anti-rabbit IgG secondary antibodies (1:10000; cat. no. AS1107; Wuhan Aspen 
Table I. Primer sequences of the genes used for RT-qPCR.

Primer sequence (5'-3')

\begin{tabular}{lll} 
Target gene & \multicolumn{1}{c}{ Forward } & \multicolumn{1}{c}{ Reverse } \\
\hline$\beta$-actin & GTCCACCGCAAATGCTTCTA & TGCTGTCACCTTCACCGTTC \\
IL-1 $\beta$ & ACGATGCACCTGTACGATCACT & GAGAACACCACTTGTTGCTCCA \\
TNF- $\alpha$ & CTCTTCTCCTTCCTGATCGTGG & CTTGTCACTCGGGGTTCGAG \\
IL-6 & TCAGCCCTGAGAAAGGAGACAT & GCTCTGGCTTGTTCCTCACTACT \\
IL-18 & TGCATCAACTTTGTGGCAATG & CTTCAAATAGAGGCCGATTTCC \\
IL-10 & AACCTGCCTAACATGCTTCG & GAGTTCACATGCGCCTTGAT \\
LIF & AGGTCTTGGCGGCAGTACAC & CCAAGGTACACGACTATGCGG \\
IL6ST & ACTTGGAGCCAGATTCCTCCT & CCCACTTGCTTCTTCACTCCA \\
IL-4 & GCAGTTCTACAGCCACCATGAG & TCTCTCTCATGATCGTCTTTAGCC \\
IL-13 & CAACATCACCCAGAACCAGAAG & GCATCCTCTGGGTCTTCTCG \\
TSG6 & GATGGATGGCTAAGGGCAGAG & CGTGTGGGTTGTAGCAATAGGC \\
& CAGCAACAATTCCTGGCGATA & GCTAAGGCGAAAGCCCTCAAT \\
\hline
\end{tabular}

IL, interleukin; TNF- $\alpha$, tumor necrosis factor $\alpha$; LIF, leukemia inhibitory factor; IL6ST, glycoprotein 130; TSG6, TNF- $\alpha$-stimulated gene 6; TGF- $\beta 1$, transforming growth factor $\beta 1$.

Biotechnology, Co., Ltd.) for $1 \mathrm{~h}$ at room temperature. Proteins were visualized using ECL Western blotting kit (Invitrogen; Thermo Fisher Scientific, Inc.) and densitometric values were analyzed using AlphaEaseFC software (AlphaInnotech, Inc. version no. 4.0).

Immunofluorescence detection. Sterile coverslips were placed on 6-well plates. Suspended cell culture solutions were added to the coverslip and incubated with $5 \% \mathrm{CO}_{2}$ for $2 \mathrm{~h}$ at $37^{\circ} \mathrm{C}$ in an incubator until cells were fixed. Cell cultures were then fixed with 4\% paraformaldehyde (cat. no. AS1018; Wuhan Aspen Biotechnology, Co., Ltd.) for $30 \mathrm{~min}$ at room temperature. After washing three times with phosphate-buffered saline (cat. no. AS1025; Wuhan Aspen Biotechnology, Co., Ltd.), the cells were incubated for $1 \mathrm{~h}$ at room temperature in PBS containing 5\% bovine serum albumin (cat. no. 10735078001; Roche Diagnostics (Shanghai), Co., Ltd.). Cells were incubated with polyclonal rabbit anti-human COX2 (1:200; cat. no. ab52237; Abcam), IL-4 (1:100; cat. no. bs-20685R; bioss), IL-10 (1:100; cat. no. ab34843; Abcam), leukemia inhibitory factor (LIF; 1:200; cat. no. ab113262; Abcam) overnight at $4^{\circ} \mathrm{C}$ followed by incubation with corresponding secondary antibodies of FITC-labeled Goat Anti-Rabbit (cat. no. AS-1110), FITC-labeled Goat Anti-Mouse (cat. no. AS-1112), CY3-labeled Goat Anti-Rabbit (cat. no. AS-1109), CY3-labeled Goat Anti-Mouse (cat. no. AS-1111), CY3-labeled Donkey Anti-Goat (cat. no. AS-1113) for $50 \mathrm{~min}$ at room temperature. All secondary antibodies were purchased from Wuhan Aspen Biotechnology, Co., Ltd. and were used at a dilution of 1:50. Cells were then washed three times with PBS for 5 min each time. DAPI staining solution was added to each well, incubated for $5 \mathrm{~min}$ at room temperature and washed three times with PBS for 5 min each time. Following this, an anti-fluorescence quencher (cat. no. AS1089; Wuhan Aspen Biotechnology, Co., Ltd.) was added to the cells and samples were viewed under a fluorescence microscope at a magnification of $\mathrm{x} 200$.

Statistical analysis. Data were analyzed using SPSS software (version 22.0; IBM Corp.). Data are presented as the mean \pm standard deviation and paired Student's t-test were used for comparisons between two groups. $\mathrm{P}<0.05$ was considered to indicate a statistically significant difference.

\section{Results}

Morphological observation of control and experimental MSCs. As presented in Fig. 1, MSCs in the control group were adherent, fibroblast-like cells, which aggregated into clusters following proliferation. MSCs cultured with CM shown suspended growth. Initially, MSCs were suspended on the surface of the CM as a single sphere; however, after $\sim 24 \mathrm{~h}$, cells aggregated spontaneously into multicellular spheroids on the surface of the CM and increased progressively (data not shown).

Effects of CM on pro- and anti-inflammatory cytokine mRNA expression in MSCs. The mRNA expression levels of pro-inflammatory genes IL-1 $\beta$, TNF- $\alpha$, IL- 6 and IL-18 were significantly decreased in the experimental group compared with the control group $\left({ }^{*} \mathrm{P}<0.05,{ }^{* *} \mathrm{P}<0.01\right.$; Fig. $\left.2 \mathrm{~A}\right)$. By contrast, the mRNA expression levels of anti-inflammatory genes TGF- $\beta 1$ were significantly increased in the experimental group compared with the control group $(\mathrm{P}<0.01$; Fig. $2 \mathrm{~B})$. The relative expression levels of IL-4 and IL-13 in the experimental group were 10-fold higher compared with the control group.

Effects of CM on pro- and anti-inflammatory cytokine protein expression in MSCs. The protein expression levels of pro-inflammatory genes IL-1 $\beta$, TNF- $\alpha$, IL- 6 and IL-18 in the 

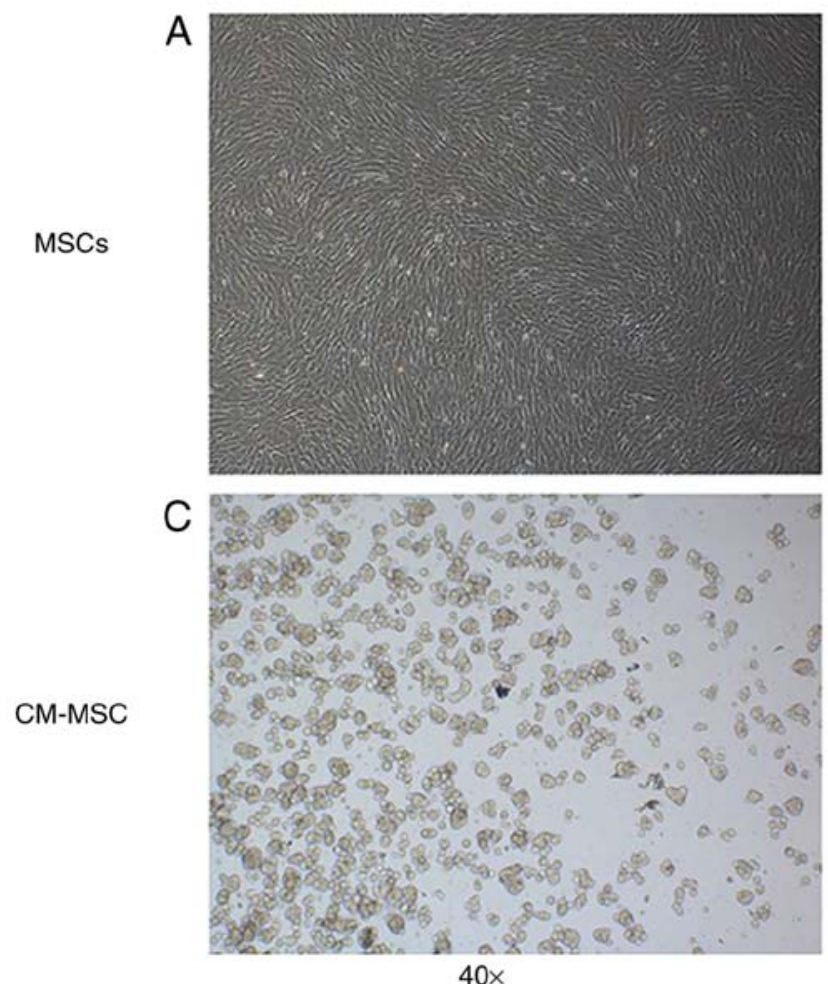

B

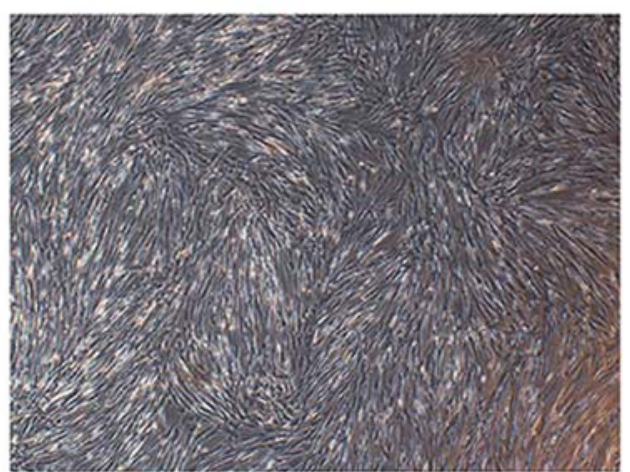

$\mathrm{D}$

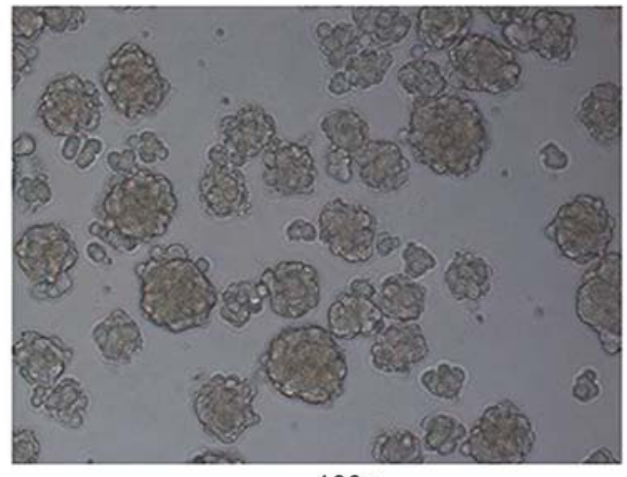

$100 x$

Figure 1. Morphological observation in control and experimental MSCs. Following $72 \mathrm{~h}$ of culturing, cell morphology in the control group at a magnification of (A) $x 40$ and (B) $\times 100$, and the experimental group at a magnification of (C) $x 40$ and (D) x100 was visualized. MSCs, mesenchymal stem cells; CM, chitosan membrane.
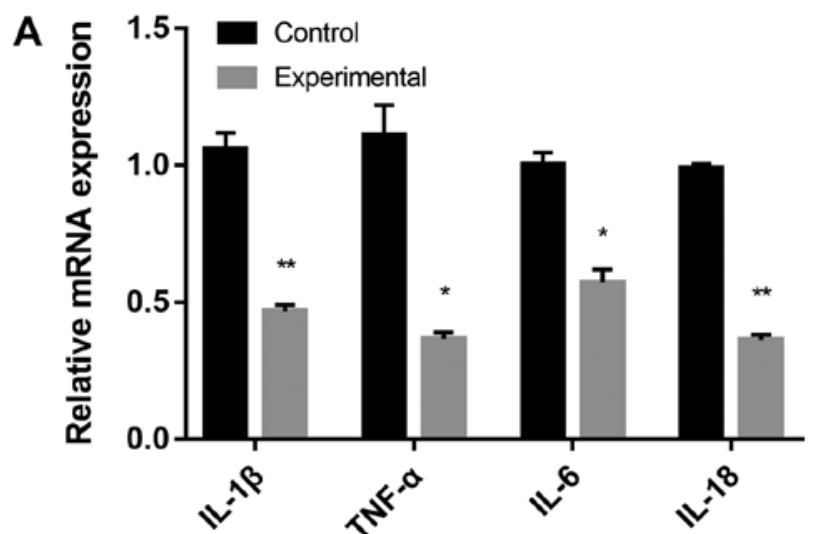

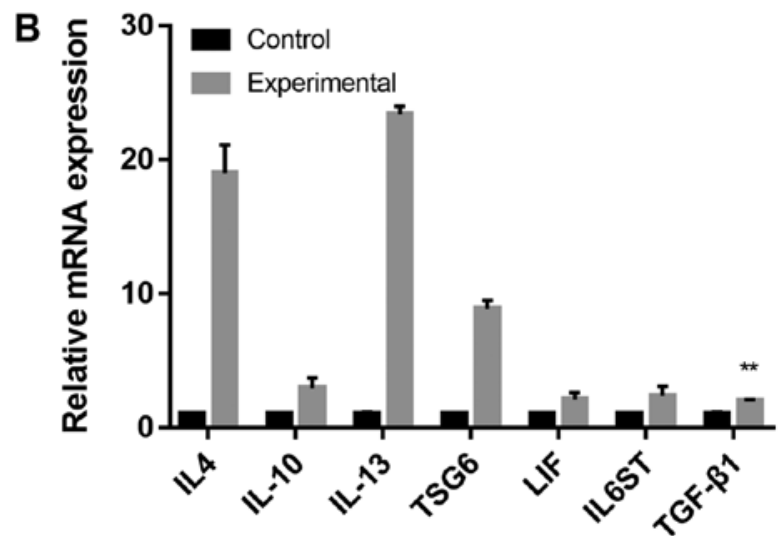

Figure 2. (A) mRNA expression levels of IL-1 $\beta$, TNF- $\alpha$, IL-6 and IL-18 in the control and experimental groups. (B) The mRNA expression levels of IL-4, IL-10, IL-13, TSG6, LIF, IL6ST and TGF- $\beta 1$ in the control and experimental groups. The control group was cultured using a traditional plate culture, while the experimental group was cultured in a plate with a CM. IL, interleukin; TNF- $\alpha$, tumor necrosis factor $\alpha$; TSG6, TNF- $\alpha$-stimulated gene 6; LIF, leukemia inhibitory factor; IL6ST, glycoprotein 130 ; TGF- $\beta 1$, transforming growth factor $\beta 1$; CM, chitosan membrane. ${ }^{*} \mathrm{P}<0.05,{ }^{* *} \mathrm{P}<0.01$.

experimental group were significantly decreased compared with the control group $\left({ }^{*} \mathrm{P}<0.05,{ }^{* *} \mathrm{P}<0.01,{ }^{* * *} \mathrm{P}<0.001\right.$; Fig. 3$)$. Furthermore, the protein expression levels of TGF- $\beta 1$ were significantly increased in the experimental group compared with the control group $(\mathrm{P}<0.01$; Fig. 3).

Immunofluorescence observations. Proteins expressed by prostaglandin synthase-2 (COX-2), IL-4, IL-10 and LIF in the experimental and control groups were located in cells and belonged to endogenous proteins (Fig. 4). The protein content of COX-2, IL-4, IL-10 and LIF in the experimental group was higher compared to the control group, indicating that the $\mathrm{CM}$ increased the expression of COX-2, IL-4, IL-10 andLIF in MSCs.

\section{Discussion}

The incidence and development of OA is characterized by the inflammatory catabolism process, which damages tissues, and the anti-inflammatory anabolism process, which protects tissues (5). Various pro- and anti-inflammatory factors mediate these processes. In OA, inflammatory cytokines interact and restrict each other to maintain the healthy metabolism of articular cartilage (1). An imbalance of inflammatory cytokines causes 


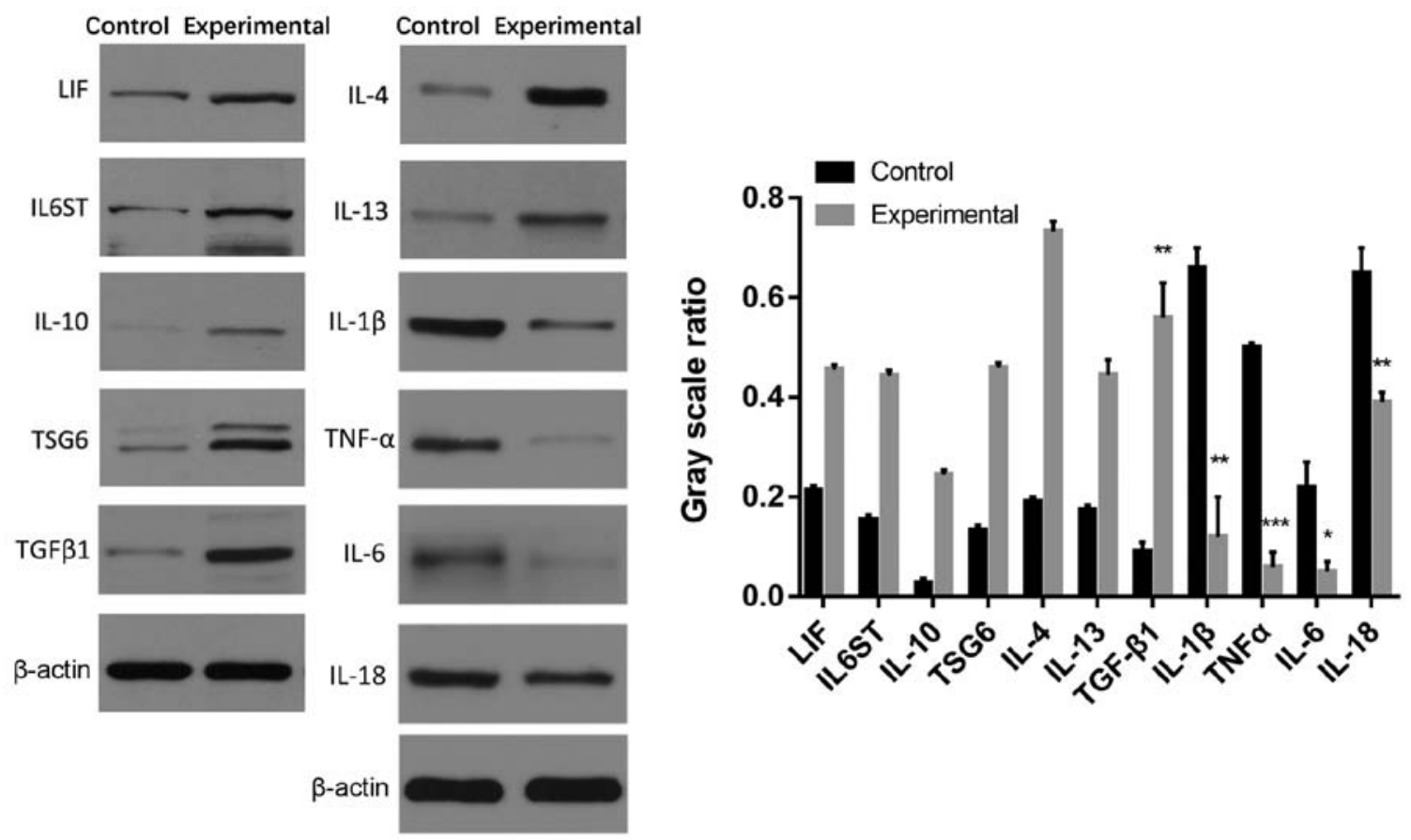

Figure 3. Protein expression of pro- and anti-inflammatory cytokines LIF, IL6ST, IL-10, TSG6, TGF- $\beta 1$, IL-4, IL- 13 , IL- $1 \beta$, TNF- $\alpha$, IL- 6 and IL-18 in the control and experimental groups. The control group was a traditional plate culture, while the experimental group was cultured in a plate with a CM. LIF, leukemia inhibitory factor; IL6ST, glycoprotein 130 ; IL, interleukin; TSG6, TNF- $\alpha$-stimulated gene 6; TGF- $\beta 1$, transforming growth factor $\beta 1$; TNF- $\alpha$, tumor necrosis factor $\alpha$; CM, chitosan membrane. ${ }^{*} \mathrm{P}<0.05,{ }^{* *} \mathrm{P}<0.01,{ }^{* * *} \mathrm{P}<0.001$.

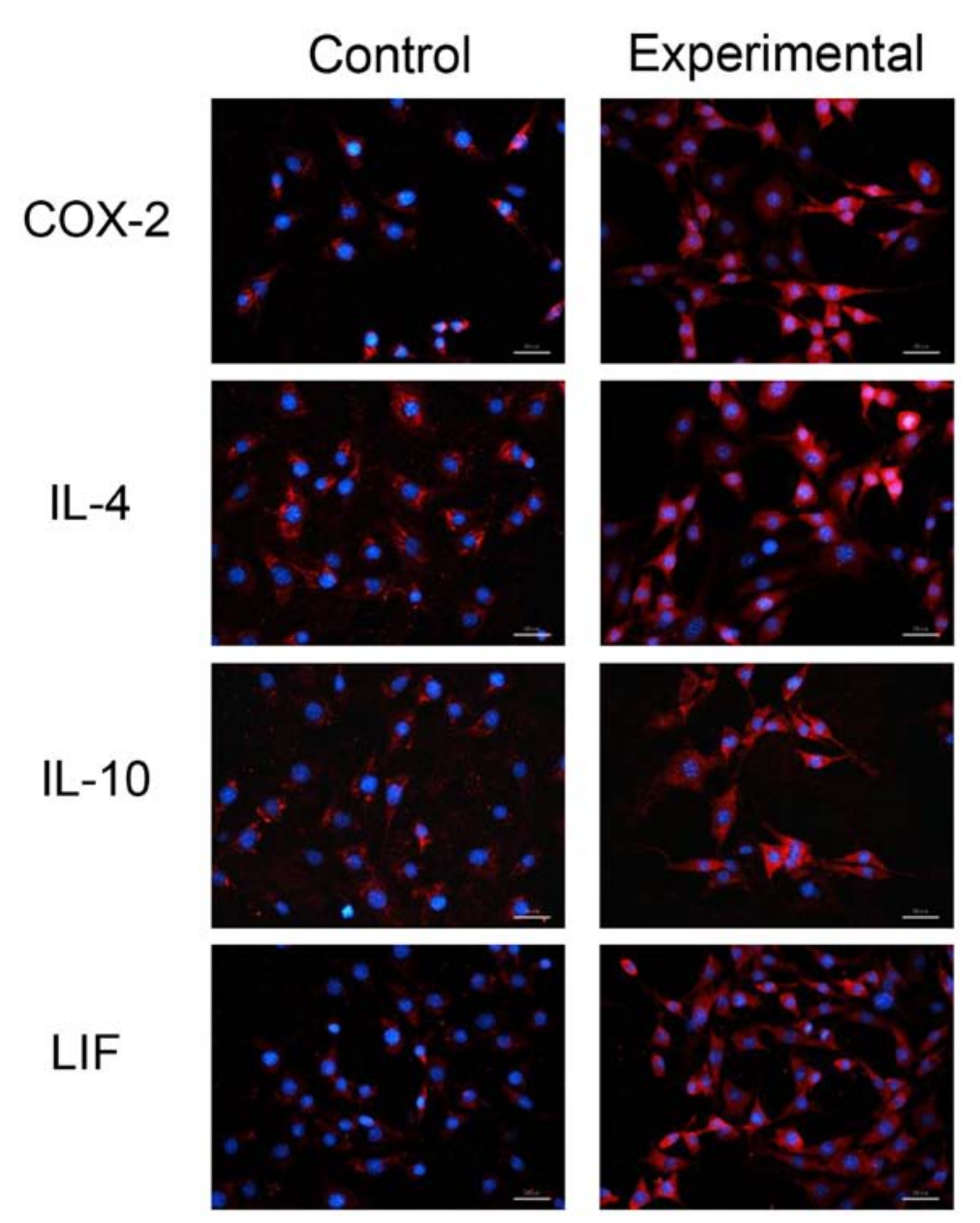

Figure 4. Distribution of COX-2, IL-4, IL-10 and LIF in MSCs in the control and experimental groups. Blue fluorescence represents nuclei. Red fluorescence represents the cytoplasm. The expression level of COX-2, IL-4, IL-10 and LIF in the experimental group was higher compared with that in the control group. Scale bars: $50 \mu \mathrm{m}$. COX-2, prostaglandin synthase-2; IL, interleukin; LIF, leukemia inhibitory factor. 
the abnormal metabolism of articular cartilage, which leads to deformation, loss and degradation of articular cartilage (3).

In the pathogenesis of OA, pro-inflammatory cytokines IL-1 $\beta$ and TNF- $\alpha$ induce a release of inflammatory mediators via the regulation of $\mathrm{NF}-\kappa \mathrm{B}$ and microtubule-associated protein kinase (MAPK) signaling pathways, causing articular cartilage degeneration, destruction and degradation. The pro-inflammatory effects of IL-1 $\beta$ and TNF- $\alpha$ in the pathogenesis of OA have been confirmed (16-18). Previous studies have demonstrated that IL- $1 \beta$ and TNF- $\alpha$ promote the absorption and degradation of articular cartilage, causing the occurrence of OA $(19,20)$. In the pathogenesis of OA, IL- $1 \beta$ and TNF- $\alpha$ initially bind to their respective receptors and regulate the release of pro-inflammatory cytokines, including IL-1 $\beta$, TNF- $\alpha$, IL-6 and IL-18 via the NF- $\kappa \mathrm{B}$ and MAPK signaling pathways (21). The expression levels of a disintegrin and metalloproteinase with thrombospondin motifs 4 (ADAMTS-4), ADAMTS-5, nitric oxide and prostaglandin $\mathrm{E}_{2}$ are upregulated and the contents of matrix metallopeptidase (MMP)-1, MMP-3 and MMP-13 are increased in chondrocytes and synovial fibroblasts, thereby enhancing the inflammatory reaction, promoting extracellular matrix (ECM) degradation, inhibiting extracellular matrix synthesis and, ultimately, leading to articular cartilage degeneration, destruction and degradation (3).

Furthermore, anti-inflammatory cytokines serve important roles in the pathogenesis of OA by inhibiting pro-inflammatory cytokines, downregulating the expression of MMPs, hindering the inflammatory response, destroying articular chondrocytes, promoting the synthesis of proteoglycans and collagen in chondrocytes, and inhibiting the progression of OA. Anti-inflammatory cytokines in OA primarily include IL-4, IL-10, IL-13, LIF, IL6ST, TSG6 and TGF- $\beta 1$ (22).

Previous studies have reported that IL-4 inhibits IL-1 and induces the release of MMP-13, thereby preventing the degradation of cartilage ECM, protecting cartilage integrity and delaying the process of OA $(23,24)$. Furthermore, IL- 4 has been revealed to inhibit the expression of ADAMTS-4 and ADAMTS-5, delay the degradation of articular cartilage ECM, and promote the synthesis of proteoglycans and collagen in chondrocytes (23). Reportedly, IL-10 inhibits the synthesis and secretion of IL-6 and other related pro-inflammatory cytokines, thus serving a role in the regulation of inflammatory immunity (25). Additionally, IL-10 inhibits the release of MMP-1, MMP-3, MMP-13 and nitric oxide in cartilage tissues, inhibits the inflammatory reaction and destruction of articular chondrocytes, and promotes the synthesis of proteoglycan and collagen in chondrocytes (23). TGF- $\beta$ is a cytokine secreted by various cells and has a regulatory effect on cell growth, differentiation and immune function (26). Previous studies have demonstrated that TGF- $\beta$ promoted the expression of metallopeptidase inhibitor-4 RNA, inhibited the activity of MMPs, reduced the formation of MMPs and protected cartilage, which delayed the development of OA $(27,28)$. Furthermore, TGF- $\beta$ promotes chondrocyte DNA synthesis, increases the number of chondrocytes and repairs inflammation-induced cartilage damage (29).

MSCs are multipotent, undifferentiated cells with extensive differentiation potential and self-replication abilities. MSCs have become a focus of interest in the treatment of $\mathrm{OA}$ as they can be easily isolated and exhibit in vitro proliferative abilities, plasticity, immunosuppressive characteristics, autocrine- and paracrine-mediated effects, migration ability to injured sites, and phenotypic stability (9). MSCs involved in tissue regeneration and can migrate to injured sites. In the local microenvironment, the directional differentiation of MSCs into specific types of functional cells directly participates in the process of tissue repair (30). Furthermore, MSCs secrete various bioactive substances, including cytokines and growth factors, which can improve microenvironment regeneration of the injured sites and inhibit local inflammation (31). Previous studies have confirmed that intra-articular injection of MSCs can be used to treat early arthritis, protect articular cartilage and increase the expression of certain anti-inflammatory-related genes and cartilage-protective factors $(32,33)$.

The survival rate of MSCs amplified by traditional adherent cultures in clinical and animal experiments is low and biological activities and therapeutic effects are poor (32). The division and differentiation of MSCs are closely associated with their microenvironment, and cytokines and proteins in the microenvironment all influence the differentiation of MSCs (34). Previous studies have demonstrated that $3 \mathrm{D}$ cell cultures can produce multicellular spheres and reproduce the microenvironment and associated physiological activities in vivo $(35,36)$. Therefore, 3D MSCs exhibit stronger biological functions and therapeutic effects compared with traditional adherent MSCs (37). The results of the present study revealed that a 3D CM culture was more effective in maintaining the anti-inflammatory properties of MSCs compared with a 2D traditional culture.

CS has high biocompatibility, no immunogenicity and low toxicity (38). The present study demonstrated that CM-cultured MSCs overcame the shortcomings of plate cultures. Similarly to the 3D culture model of hanging drop culture, CM-cultured MSCs form spheroids and exhibit enhanced transformation efficiency (39). Previous studies have reported that MSCs adhering to the CM can self-assemble to form 3D spherical cells (14). During this process, MSCs adhere and diffuse on the $\mathrm{CM}$ and reduce the number of pseudopods to form multicellular spheres. This aggregation process is different from that of suspensions or suspension cultures (13).

It has been reported that the formation of spheroids in the CM is associated with the involvement of various genes and proteins, including cadherin $(40,41)$, the Rho/Rho-associated protein kinase pathway (12) and Wnt (41). Although certain changes in gene and protein expression levels have been observed, the exact mechanism of spheroid formation in the CM remains unclear.

3D suspension cultures of MSCs form multicellular spheroids, affect the epigenetic status of MSCs and improve the anti-inflammatory properties of MSCs (12). The formation of multicellular spheres affects the expression of inflammatory cytokines in MSCs. The results of the present study demonstrated that, in the CM group, the expression levels of pro-inflammatory genes IL- $1 \beta$, TNF- $\alpha$, IL- 6 and IL-18 were significantly lower compared with the control group, and the expression levels of anti-inflammatory genes TGF- $\beta 1$ were significantly higher compared with the control group, indicating that the formation of multicellular spheres in CM-cultured MSCs was more conducive to maintaining the anti-inflammatory characteristics of MSCs.

The present study had certain limitations. Firstly, the amount of cytokines secreted by CM-cultured MSCs compared with MSCs was not examined. Secondly, cells were identified 
as MSCs by cell morphology. Further cell type identification assays would have been beneficial.

In conclusion, compared with the control group, CM-cultured MSCs formed multicellular spheres, significantly decreased the expression of pro-inflammatory cytokines and significantly increased the expression of anti-inflammatory-related genes, indicating that $\mathrm{CM}$ cultures could enhance the anti-inflammatory properties of MSCs. Although the role of pro- and anti-inflammatory cytokines in the pathogenesis of OA remains unclear, the present study provided a novel approach that may be beneficial for the OA research.

\section{Acknowledgements}

Not applicable.

\section{Funding}

The present study was funded by the National Science and Technology Support Project of Hubei (grant no. 2015BCA316) and the Science and Technology Program in Wuhan (grant no. 2016060101010045).

\section{Availability of data and materials}

The datasets used and/or analyzed during the present study are available from the corresponding author on reasonable request.

\section{Authors' contributions}

$\mathrm{XX}$ and PY reviewed literature, researched data and drafted the manuscript. PY was involved in revising the manuscript and participated in the interpretation of data. HL made contributions to the acquisition of data. BQ was responsible for the conception, design of the study and revising the manuscript critically for important intellectual content. All authors read and approved the final manuscript.

\section{Ethics approval and consent to participate}

The present study was approved by the Ethics Committee of the Renmin Hospital of Wuhan University, Wuhan, China.

\section{Patient consent for publication}

Not applicable.

\section{Competing interests}

The authors declare that they have no competing interests.

\section{References}

1. Glyn-Jones S, Palmer AJ, Agricola R, Price AJ, Vincent TL, Weinans $\mathrm{H}$ and Carr AJ: Osteoarthritis. Osteoarthritis Lancet 386: 376-387, 2015.

2. Charlier E, Deroyer C, Ciregia F, Malaise O, Neuville S, Plener Z, Malaise M and de Seny D: Chondrocyte dedifferentiation and osteoarthritis (OA). Biochem Pharmacol 165: 49-65, 2019.

3. Wojdasiewicz P, Poniatowski LA and Szukiewicz D: The role of inflammatory and anti-inflammatory cytokines in the pathogenesis of osteoarthritis. Mediators Inflamm 2014: 561459, 2014.
4. Vangsness CT Jr, Burke WS, Narvy SJ, MacPhee RD and Fedenko AN: Human knee synovial fluid cytokines correlated with grade of knee osteoarthritis - a pilot study. Bull NYU Hosp Jt Dis 69: 122-127, 2011

5. Mueller MB and Tuan RS: Anabolic/Catabolic balance in pathogenesis of osteoarthritis: Identifying molecular targets. PM R 3 (Suppl 1): S3-S11, 2011.

6. Aboushady IM, Salem ZA, Sabry D and Mohamed A: Comparative study of the osteogenic potential of mesenchymal stem cells derived from different sources. J Clin Exp Dent 10: e7-e13, 2018.

7. Gao W, Lin M, Liang A, Zhang L, Chen C, Liang G, Xu C, Peng Y, Chen C, Huang D, et al: Melatonin enhances chondrogenic differentiation of human mesenchymal stem cells. J Pineal Res 56: 62-70, 2014.

8. Du Z, Wei C, Cheng K, Han B, Yan J, Zhang M, Peng C and Liu Y: Mesenchymal stem cell-conditioned medium reduces liver injury and enhances regeneration in reduced-size rat liver transplantation. J Surg Res 183: 907-915, 2013.

9. Delling U, Brehm W, Ludewig E, Winter $\mathrm{K}$ and Jülke $\mathrm{H}$ : Longitudinal evaluation of effects of intra-articular mesenchymal stromal cell administration for the treatment of osteoarthritis in an ovine model. Cell Transplant 24: 2391-2407, 2015.

10. Saulnier N, Viguier E, Perrier-Groult E, Chenu C, Pillet E, Roger T, Maddens S and Boulocher C: Intra-articular administration of xenogeneic neonatal Mesenchymal Stromal Cells early after meniscal injury down-regulates metalloproteinase gene expression in synovium and prevents cartilage degradation in a rabbit model of osteoarthritis. Osteoarthritis Cartilage 23: 122-133, 2015.

11. Sun Y, Wang Y, Zhou L, Zou Y, Huang G, Gao G, Ting S, Lei X and Ding X: Spheroid-cultured human umbilical cord-derived mesenchymal stem cells attenuate hepatic ischemia-reperfusion injury in rats. Sci Rep 8: 2518, 2018.

12. Bartosh TJ, Ylöstalo JH, Mohammadipoor A, Bazhanov N, Coble K, Claypool K, Lee RH, Choi H and Prockop DJ: Aggregation of human mesenchymal stromal cells (MSCs) into 3D spheroids enhances their antiinflammatory properties. Proc Natl Acad Sci USA 107: 13724-13729, 2010.

13. Yeh HY, Liu BH, Sieber M and Hsu SH: Substrate-dependent gene regulation of self-assembled human MSC spheroids on chitosan membranes. BMC Genomics 15: 10, 2014.

14. Huang GS, Dai LG, Yen BL and Hsu SH: Spheroid formation of mesenchymal stem cells on chitosan and chitosan-hyaluronan membranes. Biomaterials 32: 6929-6945, 2011.

15. Livak KJ and Schmittgen TD: Analysis of relative gene expression data using real-time quantitative PCR and the 2(-Delta Delta C(T)) Method. Methods 25: 402-408, 2001.

16. Shen S, Guo J, Luo Y, Zhang W, Cui Y, Wang Q, Zhang Z and Wang T: Functional proteomics revealed IL-1 $\beta$ amplifies TNF downstream protein signals in human synoviocytes in a TNF-independent manner. Biochem Biophys Res Commun 450: 538-544, 2014

17. Ismail HM, Yamamoto K, Vincent TL, Nagase h, Troeberg L and Saklatvala J: Interleukin-1 Acts via the JNK-2 Signaling Pathway to Induce Aggrecan Degradation by Human Chondrocytes. Arthritis Rheumatol 67: 1826-1836, 2015.

18. Osta B, Roux JP, Lavocat F, Pierre M, Ndongo-Thiam N, Boivin G and Miossec P: Differential Effects of IL-17A and TNF- $\alpha$ on Osteoblastic Differentiation of Isolated Synoviocytes and on Bone Explants from Arthritis Patients. Front Immunol 6: $151,2015$.

19. Lawrence JT, Birmingham J and Toth AP: Emerging ideas: Prevention of posttraumatic arthritis through interleukin-1 and tumor necrosis factor-alpha inhibition. Clin Orthop Relat Res 469: 3522-3526, 2011.

20. Furman BD, Mangiapani DS, Zeitler E, Bailey KN, Horne PH, Huebner JL, Kraus VB, Guilak F and Olson SA: Targeting pro-inflammatory cytokines following joint injury: Acute intra-articular inhibition of interleukin-1 following knee injury prevents post-traumatic arthritis. Arthritis Res Ther 16: R134, 2014.

21. Mobasheri A, Henrotin Y, Biesalski HK and Shakibaei M: Scientific evidence and rationale for the development of curcumin and resveratrol as nutraceutricals for joint health. Int J Mol Sci 13: 4202-4232, 2012.

22. Wang T and He C: Pro-inflammatory cytokines: The link between obesity and osteoarthritis. Cytokine Growth Factor Rev 44: 38-50, 2018. 
23. Assirelli E, Pulsatelli L, Dolzani P, Platano D, Olivotto E, Filardo G, Trisolino G, Facchini A, Borzì RM and Meliconi R: Human osteoarthritic cartilage shows reduced in vivo expression of IL-4, a chondroprotective cytokine that differentially modulates IL-1 $\beta$-stimulated production of chemokines and matrix-degrading enzymes in vitro. PLoS One 9: e96925, 2014.

24. Uchimura T, Foote AT, Smith EL, Matzkin EG and Zeng L: Insulin-Like Growth Factor II (IGF-II) Inhibits IL-1 $\beta$-Induced Cartilage Matrix Loss and Promotes Cartilage Integrity in Experimental Osteoarthritis. J Cell Biochem 116: 2858-2869, 2015.

25. Fytili P, Giannatou E, Karachalios T, Malizos K and Tsezou A: Interleukin-10G and interleukin-10R microsatellite polymorphisms and osteoarthritis of the knee. Clin Exp Rheumatol 23: 621-627, 2005.

26 . Coricor $\mathrm{G}$ and Serra R: TGF- $\beta$ regulates phosphorylation and stabilization of Sox 9 protein in chondrocytes through p38 and Smad dependent mechanisms. Sci Rep 6: 38616, 2016.

27. Huang W, Mabrouk ME, Sylvester J, Dehnade F and Zafarullah M: Enhanced expression of tissue inhibitor of metalloproteinases-4 gene in human osteoarthritic synovial membranes and its differential regulation by cytokines in chondrocytes. Open Rheumatol J 5: 81-87, 2011

28. Wang X, Dong C, Li N, Ma Q, Yun Z, Cai C, An M and Ma B: Modulation of TGF $\beta$ activity by latent TGF $\beta$ binding protein 1 in human osteoarthritis fibroblast like synoviocytes. Mol Med Rep 17: 1893-1900, 2018.

29. Mueller MB, Fischer M, Zellner J, Berner A, Dienstknecht T, Prantl L, Kujat R, Nerlich M, Tuan RS and Angele P: Hypertrophy in mesenchymal stem cell chondrogenesis: Effect of TGF-beta isoforms and chondrogenic conditioning. Cells Tissues Organs 192: 158-166, 2010.

30. Spees JL, Lee RH and Gregory CA: Mechanisms of mesenchymal stem/stromal cell function. Stem Cell Res Ther 7: 125, 2016.

31. Wang S, Tong M, Hu S and Chen X: The Bioactive Substance Secreted by MSC Retards Mouse Aortic Vascular Smooth Muscle Cells Calcification. BioMed Res Int 2018: 6053567, 2018.

32. Ichiseki T, Shimasaki M, Ueda Y, Ueda S, Tsuchiya M, Souma D, Kaneuji A and Kawahara N: Intraarticularly-Injected Mesenchymal Stem Cells Stimulate Anti-Inflammatory Molecules and Inhibit Pain Related Protein and Chondrolytic Enzymes in a Monoiodoacetate-Induced Rat Arthritis Model. Int J Mol Sci 19: 203, 2018.
33. Emadedin M, Ghorbani Liastani M, Fazeli R, Mohseni F, Moghadasali R, Mardpour S, Hosseini SE, Niknejadi M, Moeininia F, Aghahossein Fanni A, et al: Long-Term Follow-up of Intra-articular Injection of Autologous Mesenchymal Stem Cells in Patients with Knee, Ankle, or Hip Osteoarthritis. Arch Iran Med 18: 336-344, 2015.

34. Liu J, Chen B, Yan F and Yang W: The Influence of Inflammatory Cytokines on the Proliferation and Osteoblastic Differentiation of MSCs. Curr Stem Cell Res Ther 12: 401-408, 2017.

35. Mehta G, Hsiao AY, Ingram M, Luker GD and Takayama S: Opportunities and challenges for use of tumor spheroids as models to test drug delivery and efficacy. J Control Release 164: 192-204, 2012.

36. Huang BW and Gao JQ: Application of 3D cultured multicellular spheroid tumor models in tumor-targeted drug delivery system research. J Control Release 270: 246-259, 2018.

37. Xu Y, Shi T, Xu A and Zhang L: 3D spheroid culture enhances survival and therapeutic capacities of MSCs injected into ischemic kidney. J Cell Mol Med 20: 1203-1213, 2016.

38. Ahsan SM, Thomas M, Reddy KK, Sooraparaju SG, Asthana A and Bhatnagar I: Chitosan as biomaterial in drug delivery and tissue engineering. Int J Biol Macromol 110: 97-109, 2018.

39. Cheng NC, Wang S and Young TH: The influence of spheroid formation of human adipose-derived stem cells on chitosan films on stemness and differentiation capabilities. Biomaterials 33: 1748-1758, 2012.

40. Hsu SH, Huang GS and Feng F: Isolation of the multipotent MSC subpopulation from human gingival fibroblasts by culturing on chitosan membranes. Biomaterials 33: 2642-2655, 2012.

41. Yeh HY, Liu BH and Hsu SH: The calcium-dependent regulation of spheroid formation and cardiomyogenic differentiation for MSCs on chitosan membranes. Biomaterials 33: 8943-8954, 2012.

This work is licensed under a Creative Commons Attribution-NonCommercial-NoDerivatives 4.0 International (CC BY-NC-ND 4.0) License. 